\title{
Exploring response signals and targets in aggressive unresectable hepatocellular carcinoma: an analysis of targeted therapy phase 1 trials
}

\author{
Ishwaria M. Subbiah ${ }^{1}$, Gerald S. Falchook ${ }^{2}$, Ahmed O. Kaseb ${ }^{3}$, Kenneth R. Hess ${ }^{4}$, \\ Apostolia M. Tsimberidou ${ }^{2}$, Siqing $\mathrm{Fu}^{2}$, Vivek Subbiah ${ }^{2}$, David S. Hong ${ }^{2}$, Aung \\ Naing ${ }^{2}$, Sarina A. Piha-Paul ${ }^{2}$, Owais Akmal ${ }^{3}$, Filip Janku² and Razelle Kurzrock ${ }^{5}$ \\ ${ }^{1}$ Division of Cancer Medicine, University of Texas MD Anderson Cancer Center, Houston, TX, USA \\ ${ }^{2}$ Department of Investigational Cancer Therapeutics, University of Texas MD Anderson Cancer Center, Houston, TX, USA \\ ${ }^{3}$ Department of Gastrointestinal Medical Oncology, University of Texas MD Anderson Cancer Center, Houston, TX, USA \\ ${ }^{4}$ Department of Biostatistics, University of Texas MD Anderson Cancer Center, Houston, TX, USA \\ ${ }^{5}$ Moores Cancer Center, University of California San Diego, San Diego, CA, USA \\ Correspondence to: Ishwaria M. Subbiah, email: isubbiah@mdanderson.org \\ Keywords: targeted agents, novel therapeutics, management, systemic therapy, clinical trials \\ Received: June 01,2015 Accepted: June 11,2015 Published: June 23, 2015
}

This is an open-access article distributed under the terms of the Creative Commons Attribution License, which permits unrestricted use, distribution, and reproduction in any medium, provided the original author and source are credited.

\section{ABSTRACT}

PURPOSE: Patients with advanced hepatocellular carcinoma (HCC) have limited effective therapeutic options. Given the rapid advanced in drug development and emergence of novel agents, we analyzed the characteristics and outcomes of HCC patients treated on early phase trials with an emphasis on targeted therapies.

METHODS: We reviewed the records of consecutive HCC patients evaluated in the Phase I Clinical Trials Program at MD Anderson from March 2004.

RESULTS: Thirty-nine patients were not treated due to poor performance status $(n=22,56 \%)$ and decision to pursue alternate therapies $(n=10,27 \%)$. Of 61 treated patients (median age, 60 years; median prior therapies, 3 ), eight patients $(13 \%)$ attained stable disease lasting $\geq 6$ months; four $(7 \%)$ had a partial response, mainly with anti-angiogenic or multikinase inhibitors. Median Phase I progressionfree survival (PFS) was 2.6 months versus 4.4 months ( $p$ 0.019) and 4.1 months ( $p$ 0.27) for their first-, and second-line FDA-approved therapy. Molecular analysis showed frequent PTEN loss (10/19 patients, 53\%) and P53 mutation (4/4 patients tested). On multivariate analysis, independent factors predicting shorter survival were white ethnicity/race ( $p 0.031)$, cirrhosis $(p 0.016)$, and serum sodium $(p 0.0013)$.

CONCLUSIONS: In our heavily-pretreated HCC patients, the phase I PFS was comparable to that of $2^{\text {nd }}$-line therapy, highlighting a potential role for clinical trials after progression on first-line therapy. The response rate (SD>6 months/PR) of $20 \%$ was observed with early signals of activity in regimens combining inhibitors of angiogenesis, multiple kinases and mTOR with preliminary molecular analysis revealing prevalence of PTEN loss.

\section{INTRODUCTION}

Advanced hepatocellular carcinoma (HCC) remains a therapeutic challenge with limited effective treatment options. Patients with limited localized disease are considered for surgical resection or liver transplantation, both of which remain the only potentially curative options. [1-3] However in patients with recurrent or unresectable disease, the mainstay of therapy remains monotherapy with sorafenib, an oral multikinase inhibitor of vascular endothelial growth factor (VEGF), platelet derived growth factor receptor (PDGFR) and RAF.[4, 5] The pivotal multicenter, phase 3, double-blind, placebo-controlled 
trial in 602 patients with advanced HCC demonstrates an improvement in the median overall survival (10.7 months) in the sorafenib group versus the placebo group (7.9 months).[6] Multimodal therapeutic approaches using arterial embolization or chemoembolization also demonstrate a survival benefit over supportive care alone in patients with unresectable disease.[7, 8] Recent advancements incorporated the use of radioembolization with yttrium-90 into the treatment algorithm for intermediate to advanced HCC with Child-Pugh class A or B.[9-11] With the expanding development of agents targeting angiogenesis and aberrant cell signaling, we explored the characteristics and outcomes of patients with advanced HCC who received treatment on a phase I clinical trial with an emphasis on targeted therapies.

\section{RESULTS}

\section{Patients characteristics}

Of the 100 patients with HCC referred to the Phase I Clinical Trials program, 39 patients were not enrolled in a phase I clinical trial due to poor performance status (ECOG $>3, N=22,56 \%$ ), decision to pursue alternate therapies including treatments closer to home $(N=10$, $26 \%)$, no evidence of disease post-resection $(N=3)$, prohibitive lab abnormalities $(N=2)$ and insurance denial $(N=2)$.

Overall, 61 patients who participated in a phase I trial are included henceforth in this analysis. Pretreatment characteristics at presentation to the Phase I clinic are summarized in Table 1. The median age at diagnosis was 60 years (range, 11-84 years). There were 12 women and 49 men of whom 30 (49\%) were White, 11 (18\%) Asian, $10(16 \%)$ African American, and 10 (16\%) of Hispanic origin. Eleven patients (18\%) had an ECOG PS of 0, 49 patients $(80 \%)$ had a PS of 1 and $1(2 \%)$ patient a 2 . The median number of metastatic sites was 2 (range 0-5). The most common sites of metastases at time of Phase I referral were liver $(N=49,80 \%$ of patients $)$, lymph nodes $(N=31,51 \%)$, lung $(N=25,41 \%)$, peritoneum $(N=14$, $23 \%)$, bone $(N=13,21 \%)$, adrenal $(N=8,13 \%)$, and other (ovary, pancreas, spleen; $N=3,5 \%$ ). Regarding the extent of liver disease, 41 patients $(37 \%)$ were classified as modified Child-Pugh class A while the remaining 20 (33\%) had Class B disease.

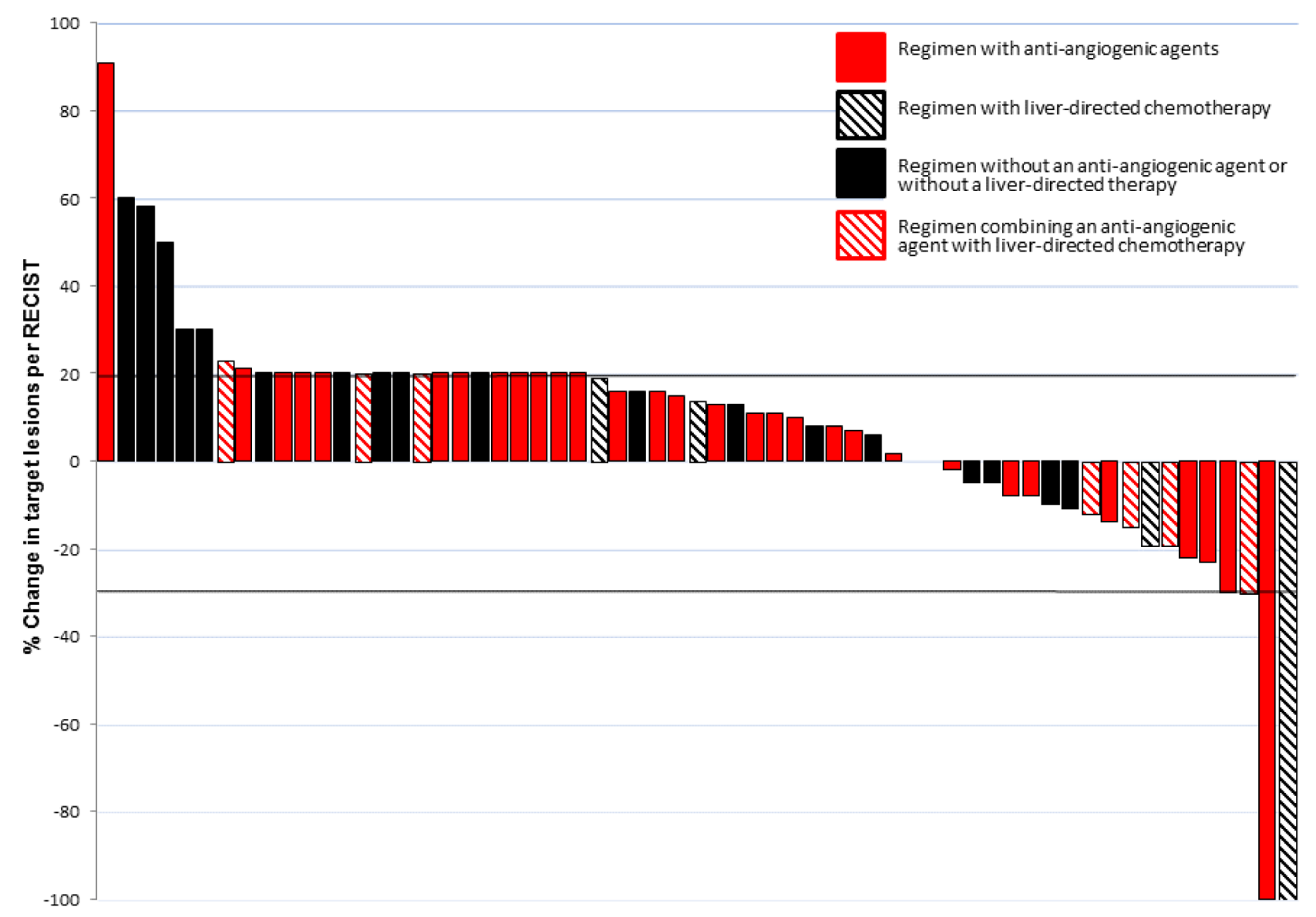

Figure 1: Waterfall plot showing the best responses to phase I therapy per RECIST. 
Table 1: Patient characteristics

\begin{tabular}{|c|c|c|}
\hline & $\mathbf{N}$ & $\%$ \\
\hline \multicolumn{3}{|l|}{ Gender } \\
\hline Male & 49 & $80 \%$ \\
\hline Female & 12 & $20 \%$ \\
\hline \multicolumn{3}{|l|}{ Age at time of diagnosis } \\
\hline \begin{tabular}{|l|l} 
Median (range) \\
\end{tabular} & 60.2 & $(11.3-83.5)$ \\
\hline \multicolumn{3}{|l|}{ Race/Ethnicity } \\
\hline \begin{tabular}{l|l} 
& White \\
\end{tabular} & 30 & $49 \%$ \\
\hline Asian & 11 & $18 \%$ \\
\hline Hispanic & 10 & $16 \%$ \\
\hline African American & 10 & $16 \%$ \\
\hline \multicolumn{3}{|l|}{ ECOG Performance Status } \\
\hline \begin{tabular}{l|l} 
& 0 \\
\end{tabular} & 11 & $18 \%$ \\
\hline 1 & 49 & $80 \%$ \\
\hline 2 & 1 & $2 \%$ \\
\hline \multicolumn{3}{|l|}{ Underlying liver pathology } \\
\hline \begin{tabular}{l|l} 
& Hepatitis C \\
\end{tabular} & 20 & $33 \%$ \\
\hline Alcohol abuse & 16 & $26 \%$ \\
\hline Hepatitis B & 20 & $33 \%$ \\
\hline Steatohepatitis & 11 & $18 \%$ \\
\hline Autoimmune hepatitis & 3 & $5 \%$ \\
\hline Hemochromatosis & 1 & $2 \%$ \\
\hline None & 7 & $11 \%$ \\
\hline \multicolumn{3}{|l|}{ Comorbidities } \\
\hline \begin{tabular}{l|l} 
& Diabetes \\
\end{tabular} & 16 & $26 \%$ \\
\hline Hyperlipidemia & 9 & $15 \%$ \\
\hline Coronary artery disease & 8 & $13 \%$ \\
\hline \multicolumn{3}{|l|}{ Imaging characteristics } \\
\hline \begin{tabular}{l|l} 
& Presence of cirrhosis
\end{tabular} & 30 & $49 \%$ \\
\hline Presence of ascites & 21 & $34 \%$ \\
\hline Portal hypertension & 17 & $28 \%$ \\
\hline Portal vein thrombosis & 29 & 48 \\
\hline Lobar distribution & & \\
\hline Unilobar & 25 & $41 \%$ \\
\hline Bilobar & 36 & $59 \%$ \\
\hline Distribution & & \\
\hline Solitary & 6 & $10 \%$ \\
\hline Multifocal & 55 & $90 \%$ \\
\hline \# of metastatic sites, median (range) & 2 & $(0-5)$ \\
\hline \multicolumn{3}{|l|}{ Metastatic sites } \\
\hline \begin{tabular}{l|l} 
& Liver \\
\end{tabular} & 49 & \\
\hline Lymph nodes & 31 & \\
\hline Lung & 25 & \\
\hline Peritoneum & 14 & \\
\hline Bone & 13 & \\
\hline Adrenal & 8 & \\
\hline Other & 3 & \\
\hline
\end{tabular}


Table 2: Characteristics of responses (PR $+\mathrm{SD}>6$ months)

\begin{tabular}{|c|c|c|c|c|c|}
\hline Pt \# & Age/Sex & Phase I Regimen & RECIST response & $\begin{array}{l}\% \text { change } \\
\text { in target } \\
\text { lesions per } \\
\text { RECIST }\end{array}$ & $\begin{array}{l}\text { Phase I } \\
\text { PFS (m) }\end{array}$ \\
\hline 1 & $54 / \mathrm{M}$ & Bevacizumab + sorafenib & SD & -8 & 9.5 \\
\hline 2 & $63 / \mathrm{F}$ & $\begin{array}{l}\text { HAI Oxaliplatin + IV 5FU, LV + } \\
\text { bevacizumab }\end{array}$ & SD & -15 & 11.8 \\
\hline 3 & $74 / \mathrm{F}$ & Bevacizumab + sorafenib & SD & -14 & 10.4 \\
\hline 4 & $56 / \mathrm{F}$ & Regorafenib & $\begin{array}{l}\text { PR (CR of } \\
\text { lesions; stable bone } \\
\text { mets) }\end{array}$ & -100 & $27.0+$ \\
\hline 5 & $56 / \mathrm{M}$ & Regorafenib & SD & 10 & 7.8 \\
\hline 6 & $73 / \mathrm{M}$ & $\begin{array}{l}\text { Novel inhibitor of HDAC, } \\
\text { EGFR1, and Her2 }\end{array}$ & SD & 6 & 11.1 \\
\hline 7 & $57 / \mathrm{M}$ & HAI paclitaxel & $\begin{array}{lrr}\text { PR (CR of } & \text { target } \\
\text { lesions; } & \text { stable } & \text { non- } \\
\text { target } & \text { previously } \\
\text { embolized } & \text { liver } \\
\text { lesions) } & \\
\end{array}$ & -100 & 41.3 \\
\hline 8 & 49/M & $\begin{array}{l}\text { HAI Oxaliplatin + IV 5FU, LV + } \\
\text { bevacizumab }\end{array}$ & PR & -30 & 6.8 \\
\hline 9 & $58 / \mathrm{M}$ & Regorafenib & SD & 8 & 7.5 \\
\hline 10 & $23 / \mathrm{M}$ & Bevacizumab + sorafenib & SD & -23 & 7.4 \\
\hline 11 & $46 / \mathrm{F}$ & Pazopanib + everolimus & PR & -30 & 8.3 \\
\hline 12 & $68 / \mathrm{M}$ & Bevacizumab + bortezomib & SD & 2 & 6.2 \\
\hline
\end{tabular}

Abbreviations: EGFR epidermal growth factor receptor, FU fluorouracil, HAI hepatic arterial infusion, LV leucovorin.

\section{Therapy before enrollment on phase I trials}

Overall, of the 61 patients enrolled on a phase I trial, four patients had received no prior therapy because of the unavailability of reasonable, conventional therapy for the extent of their disease or patient desire to pursue clinical trial. The remaining 57 patients had a median of 3 prior systemic therapies before referral to the phase I clinic (range, $1-8)$. Twenty six patients $(43 \%)$ also underwent a prior chemoembolization, $17(28 \%)$ a surgical resection, 12 (20\%) yttrium-90 radioembolization, $12(20 \%)$ radiation, $8(12 \%)$ a radiofrequency ablation, and $3(5 \%)$ an orthotopic liver transplantation.

For their first-line treatment in the advanced/ metastatic setting, 41 of 61 patients received a sorafenibbased regimen, of whom 39 received single-agent sorafenib while 2 received sorafenib in combination with erlotinib. Three patients received erlotinib plus bevacizumab; 5 received a capecitabine-based regimen, 4 received a gemcitabine-based regimen, 3 received platinum combination therapy, and 1 received experimental therapy on phase II trial with a novel camptothecin analog. 33 patients did not receive a second line treatment with FDA-approved agents and instead proceeded with a phase I clinical trial.

\section{Treatment}

Overall, patients were initially treated on 1 of 31 different phase I clinical trials; of these trials, 19 patients received therapy on 11 "first-in-human" trials with novel targeted inhibitors against VEGFR, kit, cMET, EGFR, HER2, CHK1, RAS, organic arsenic or an oleander extract derivative.

Of 61 patients, $39(64 \%)$ were treated on a trial with combination therapy of two or more agents while 22 patients $(36 \%)$ received treatment with a single agent. Ten (16\%) patients received liver-directed treatment with direct infusion of a cytotoxic agent into the hepatic artery, of which 2 as a single agent and 8 in combination most commonly with bevacizumab. $37(61 \%)$ received an oral or intravenous angiogenesis inhibitor, $6(10 \%)$ of whom received the anti-angiogenic agent in combination with liver-directed chemotherapy. Forty-nine patients $(80 \%)$ received targeted agent(s) alone, nine (15\%) received targeted agent(s) in combination with cytotoxic chemotherapy, and three (5\%) received cytotoxic chemotherapy alone. The median number of cycles 
Table 3: Summary of univariate analysis

\begin{tabular}{|c|c|c|c|c|}
\hline & \multicolumn{2}{|c|}{ Association with PFS } & \multicolumn{2}{|c|}{ Association with OS } \\
\hline & \begin{tabular}{|l|} 
Hazard ratio \\
$(95 \%$ CI $)$
\end{tabular} & $P$-value & $\begin{array}{l}\text { Hazard ratio } \\
(95 \% \mathrm{CI})\end{array}$ & $P$-value \\
\hline Gender & $0.7(0.4,1.4)$ & 0.31 & $0.8(0.3,1.7)$ & 0.51 \\
\hline Age at diagnosis & $1.3(0.8,2.1)$ & 0.35 & $1.6(0.9,2.9)$ & 0.14 \\
\hline ECOG PS 0 vs. $1-2$ & $0.8(0.4,1.5)$ & 0.44 & $1.0(0.5,2.1)$ & 0.95 \\
\hline $\begin{array}{l}\text { Ethnicity/Race } \\
\text { (White vs. non-white) }\end{array}$ & $1.2(0.7,2.0)$ & 0.47 & $2.0(1.0,3.9)$ & 0.039 \\
\hline \multicolumn{5}{|c|}{ Risk factors for chronic liver disease } \\
\hline Alcohol abuse & $0.9(0.5,1.6)$ & 0.66 & $0.6(0.3,1.2)$ & 0.12 \\
\hline Hepatitis C & $1.0(0.6,1.8)$ & 0.99 & $0.7(0.4,1.5)$ & 0.38 \\
\hline Hepatitis B & $1.2(0.7,2.2)$ & 0.47 & $1.5(0.8,2.9)$ & 0.2 \\
\hline \multicolumn{5}{|l|}{ Other comorbidities } \\
\hline Coronary artery disease & $1.6(0.7,3.4)$ & 0.27 & $1.7(0.7,4.4)$ & 0.3 \\
\hline Type II diabetes & $0.7(0.4,1.3)$ & 0.24 & $0.8(0.4,1.7)$ & 0.6 \\
\hline \multicolumn{5}{|c|}{ Extent of chronic liver disease } \\
\hline \begin{tabular}{l|l} 
Cirrhosis & \\
\end{tabular} & $1.9(1.1,3.2)$ & 0.016 & $2.2(1.2,4.0)$ & 0.012 \\
\hline Portal hypertension & $2.2(1.2,4.0)$ & 0.011 & $2.2(1.1,4.4)$ & 0.038 \\
\hline Portal vein thrombosis & $1.1(0.7,1.8)$ & 0.69 & $1.2(0.6,2.2)$ & 0.57 \\
\hline Ascites & $2.1(1.2,3.6)$ & 0.012 & $2.4(1.2,4.5)$ & 0.013 \\
\hline \multicolumn{5}{|l|}{ Extent of HCC } \\
\hline \# of metastatic sites & $1.2(0.7,2.0)$ & 0.47 & $1.5(0.8,2.8)$ & 0.19 \\
\hline \# of prior therapies & $0.8(0.5,1.4)$ & 0.51 & $1.0(0.5,1.8)$ & 0.96 \\
\hline \multicolumn{5}{|l|}{ Baseline laboratory values } \\
\hline Anemia $(\mathrm{Hg}<10.5 \mathrm{~g} / \mathrm{dL})$ & $0.8(0.4,1.6)$ & 0.6 & $1.3(0.6,2.8)$ & 0.57 \\
\hline Elevated LDH (>618IU/L) & $1.4(0.8,2.4)$ & 0.23 & $1.9(1.0,3.5)$ & 0.051 \\
\hline $\begin{array}{l}\text { Thrombocytopenia }(<158 \mathrm{~K} / \\
\text { UL) }\end{array}$ & $0.9(0.6,1.6)$ & 0.8 & $1.1(0.6,2.0)$ & 0.8 \\
\hline $\begin{array}{l}\text { Elevated alpha-fetoprotein } \\
(>120)\end{array}$ & $1.2(0.7,2.0)$ & 0.5 & $1.5(0.8,2.8)$ & 0.22 \\
\hline $\begin{array}{l}\text { Elevated total bilirubin } \\
(>1 \mathrm{mg} / \mathrm{dL})\end{array}$ & $1.5(0.8,2.7)$ & 0.22 & $2.3(1.2,4.6)$ & 0.025 \\
\hline Elevated INR $(>1.1)$ & $1.5(0.9,2.6)$ & 0.1 & $1.4(0.8,2.7)$ & 0.23 \\
\hline Elevated ALT $(>56 \mathrm{IU} / \mathrm{L})$ & $1.0(0.6,1.8)$ & 0.97 & $0.8(0.4,1.5)$ & 0.45 \\
\hline Elevated AST $(>46 \mathrm{IU} / \mathrm{L})$ & $1.5(0.7,3.0)$ & 0.26 & $2.0(0.7,5.5)$ & 0.16 \\
\hline $\begin{array}{ll}\text { Alkaline } & \text { phosphatase } \\
>126 \mathrm{IU} / \mathrm{L} & \end{array}$ & $1.5(0.8,2.9)$ & 0.24 & $1.6(0.7,3.5)$ & 0.24 \\
\hline Abnormal serum sodium & $2.8(1.2,6.4)$ & 0.028 & $3.0(1.2,7.4)$ & 0.031 \\
\hline Serum albumin $<3.5 \mathrm{~g} / \mathrm{dL}$ & $2.8(1.5,5.1)$ & 0.0013 & $5.2(2.4,11.2)$ & $<0.0001$ \\
\hline Serum creatinine $>1.3 \mathrm{mg} / \mathrm{dL}$ & NR & $\mathrm{n} / \mathrm{a}$ & NR & $\mathrm{n} / \mathrm{a}$ \\
\hline
\end{tabular}

Abbreviations: ALT Alanine aminotransferase; AST Aspartate aminotransferase; ECOG Eastern Cooperative Oncology Group; LDH Lactate dehydrogenase; NR not reached; PS Performance status. 
Table 4: Summary of multivariate analysis

\begin{tabular}{|l|l|l|l|l|}
\hline & $\begin{array}{l}\text { Hazard } \\
\text { ratio }\end{array}$ & $\begin{array}{l}\text { Lower } \\
\text { limit }\end{array}$ & $\begin{array}{l}\text { Upper } \\
\text { limit }\end{array}$ & P value \\
\hline \multicolumn{5}{|l|}{ Analytic variable associated with shorter PFS } \\
\hline Cirrhosis & 1.8 & 1 & 3.1 & 0.038 \\
\hline Portal hypertension & 2.2 & 1.2 & 4.2 & 0.017 \\
\hline Abnormal serum sodium & 4.3 & 1.8 & 10.3 & 0.0012 \\
\hline Analytic variable associated with shorter OS \\
\hline White race & 2.2 & 1.1 & 4.6 & 0.031 \\
\hline Cirrhosis & 2.3 & 1.2 & 4.4 & 0.016 \\
\hline Abnormal serum sodium & 4.9 & 1.9 & 13 & 0.0013 \\
\hline
\end{tabular}

Abbreviations: OS overall survival; PFS progression-free survival.

received was two (range $1-28$ ). Eighteen patients went on to receive therapy under a second phase I trial; of these patients, 11 received treatment on 3 phase I trials, 2 were treated on a $4^{\text {th }}$ trial.

\section{Response}

Fourteen patients were not restaged prior to end of cycle 2 due to clinical deterioration and early disease progression and are identified on the waterfall plot as having a $20 \%$ increase in tumor size (Figure 1). Of the 61 patients treated on studies, $4(7 \%)$ had a partial response (PR), 23 had stable disease (38\%) including 8 (13\%) who had $\mathrm{SD}>6$ months, with a SD $>6$ months/PR rate of $20 \%$ (12 of 61 patients); 10 of these 12 patients received an oral or IV inhibitor of angiogenesis. The characteristics and specific treatment regimens of these responders are detailed in Table 2. Thirty-five patients (57\%) had progressive disease (PD). The highest response rates (SD $>6$ months/PR) were observed in patients treated with protocols that included agents targeting angiogenesis and mTOR. Of 10 patients who were treated on an HAIbased protocol, $3(30 \%)$ has a significant clinical response including two with prolonged $\mathrm{SD}>6$ months and $1 \mathrm{PR}$.

One dramatic response was observed in a 56year old Asian woman with metastatic HCC in setting of hepatitis C, Child-Pugh class A. Her prior therapies included sorafenib monotherapy and then a combination of bevacizumab plus erlotinib. Upon progression, she then enrolled on phase I therapy with regorafenib, then a novel oral small molecular inhibitor of VEGFR2 and 3, and Ret, Kit, PDGFR and Raf kinases. She demonstrated a complete response of target lesions with stable nonmeasurable bone lesions, hence deemed a partial response per RECIST, and has maintained this remarkable response for 27 months ongoing. A second response was observed in a 57-year old man who received HAI paclitaxel; he demonstrated a complete response of target liver lesions with stable post-embolization non-target hepatic masses, thereby attaining a partial response.

\section{Survival and toxicities}

The median time from diagnosis of advanced/ metastatic disease to date of primary evaluate in the phase I clinic was 9.6 months for the 61 patients who enrolled on a phase I trials. The median time from the primary phase I consultation to beginning therapy on a phase I clinical trial was 19 days. The median overall survival from day one on a phase I trial was 7 months $(95 \%$ CI 6, 13). Median PFS for 61 treated patients was 2.6 months (95\% CI 1.9, 3.4) on phase I clinical trials. Among these 61 patients, the median PFS on their first-line and second-line prior therapies with FDA approved agents given in the advanced setting was 4.4 months and 4.1 months, respectively. In comparison, the PFS on the first line FDA-approved therapy given in the advanced setting prior to Phase I referral was improved compared to the PFS on phase I therapy $(p 0.019)$. However, the second-line therapy with FDA-approved drugs was comparable to the PFS on a phase I trial ( $p$ 0.27). The PFS on first-, second-line and Phase I therapy are shown in Figure 2.

Among the 61 treated patients, 42 (69\%) had died at the time of analysis. The 90-day mortality was $38 \%$ with 38 patients alive at 3 months after beginning phase I therapy; the 6-month mortality was $61 \%$ with 24 patients alive at 6 months after beginning therapy on phase I trials. Importantly, there was no treatment-related mortality. One patient treated on a combination regimen that included sorafenib experienced grade 3 hand foot syndrome that was not responsive to a dose reduction. This patient ultimately showed disease progression on restaging imaging. A second patient developed a mild headache, dizziness and left-sided visual field blurriness five days after beginning a sunitinib-based combination therapy and was found to have a small right parieto-occipital intracranial hemorrhage, possibly related to therapy. There were no other high-grade toxicities reported. 


\section{Prognostic factors for survival}

We conducted univariate and multivariate analysis to evaluate the effects on survival of variables including age, sex, race/ethnicity, ECOG performance status, risk factors for liver disease (alcohol abuse, hepatitis C, hepatitis B), comorbidities (coronary artery disease, type II diabetes), extent of liver disease (presence of cirrhosis, portal hypertension, ascites, portal vein thrombosis), alpha-fetoprotein; history of thromboembolism; number of prior therapies; presence of liver metastases; number of metastatic sites; hemoglobin level; platelet count; and albumin, lactate dehydrogenase (LDH), alkaline phosphatase, bilirubin, alanine aminotransferase, aspartate aminotransferase, sodium, and creatinine levels (Table 3). Predictors of shorter Phase I PFS in univariate analysis were the presence of cirrhosis $(p 0.016)$, portal hypertension ( $p$ 0.011), ascites ( $p 0.012$ ), abnormal sodium ( $p 0.028)$ and hypoalbuminemia $(0.0013)$; these five factors also predicted for shorter overall survival ( $p$ $0.012,0.038,0.013,0.031$, and $<0.0001$ respectively).
The multivariate analysis showed cirrhosis ( $p$ 0.038), portal hypertension $(p<0.017)$, and serum sodium $(p$ 0.0012 ) as predictors of shorter PFS on phase I therapy while cirrhosis, white race and abnormal serum sodium predicted for a shorter OS (Table 4).

\section{Molecular analysis}

Testing for mutations in KRAS, NRAS, BRAF, CKIT, EGFR, PIK3CA, TP53, MET, GNAQ, AKT1, $A L K$ rearrangement as well as immunohistochemistry for PTEN loss, ER, and ALK1 and HER-2/neu and MET amplification via FISH was completed on patients with adequate available tissue in the MD Anderson CLIAcertified laboratories. On immunohistochemistry, 10 of 19 tested patients demonstrated an abnormality in PTEN expression with 7 tumors showing frank PTEN loss and 3 samples showing very faint PTEN expression $(<10 \%$ of stained cells). PCR-based DNA sequencing analysis was performed in exons 4 to 9 of the TP53 gene. The lower limit of detection is approximately one cell bearing

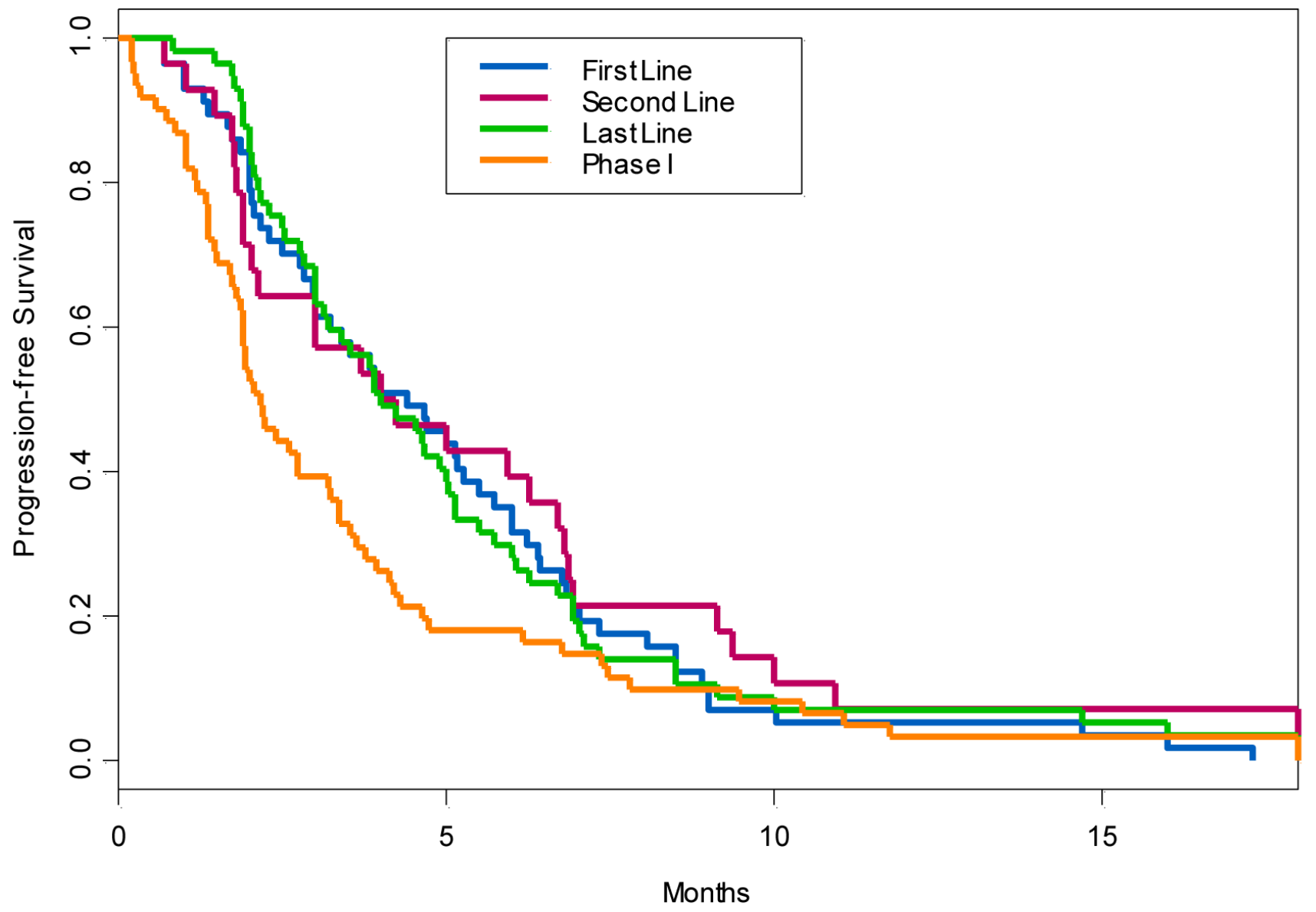

Figure 2: Progression-free survival of patients treated on phase I trials when compared to their first-line, second-line and last systemic antitumor therapy given in advanced setting prior to phase I referral. 
the mutation per five cells $(20 \%)$. All 4 tested patients demonstrated a TP53 aberration, one patient with a 12 base pair deletion in exon 4 and 3 patients with a missense mutation in codon 177 in exon 5 (P177L), codon 158 in exon $5(\mathrm{R} 158 \mathrm{H})$, and codon 272 in exon 8 (V272M). KIT, $P I K 3 C A$, and $M E T$ mutations were found in individual patients. The remainder of the mutational analyses was negative including KRAS (all 19 tested), PIK3CA (17 of 18 tested), $B R A F$ (all 17 tested), and $E G F R$ (all 13 tested).

\section{DISCUSSION}

Unresectable hepatocellular carcinoma in the setting of Child-Pugh class A or B liver disease remains a clinical challenge particularly after relapse on sorafenib given in the metastatic setting. Alternate recommended options include locoregional therapy with transarterial chemoembolization, conformal or stereotactic radiation, or clinical trial.[13] Multimodal approaches include liver-directed therapies such as embolization with the radionuclide yttrium-90.[14] However, for the patient with extrahepatic disease, the need for novel targeted systemic therapies and combination regimen is self-evident.

In our analysis, patterns of early signals suggesting clinical activity emerged in regimens that target angiogenesis particularly in combination with multikinase inhibition using sorafenib. Of particular interest is the observation of clinical response using a combination of bevacizumab and sorafenib in our HCC patients who have all previously received single-agent sorafenib. These patients had demonstrated disease regression with sorafenib monotherapy given as frontline agent in the metastatic setting but subsequent development of secondary resistance with disease progression. This concept of successful retreatment after initial response and subsequent progression has been explored in small case series and provides an intriguing challenge to the prevailing convention that a resistant tumor is unlikely to respond to a rechallenge with a similar regimen. $[15,16]$ Additional response signals were observed in regimens utilizing intravenous angiogenesis inhibition in combination with liver-directed infusion of cytotoxic chemotherapy. Indeed ten of the twelve prolonged responders received an angiogenesis inhibitor, of which two were in combination with hepatic arterial infusion therapy.

For the clinician faced with the relapsed advanced HCC patient, the timing of referral for a clinical trial remains essential point of decision. In our analysis, frontline therapy with sorafenib given in the advanced setting prior to phase I referral had an improved PFS in comparison to the PFS in phase I clinical trial. However the PFS on the second line therapy with FDAapproved agents given in the advanced setting prior to phase I referral was comparable to the PFS on phase I trial. Furthermore, in our analysis, of the 100 patients referred for phase I trials, 39\% were ineligible mainly due to decline in performance status (ECOG PS of 3 or greater) and prohibitively abnormal laboratory values, underscoring the importance of early participation in clinical trials after progression on frontline therapy in the metastatic setting.

Overall, the pattern of molecular aberrations seen in HCC is slowly emerging. In our analysis, the most common molecular aberration was observed in the expression of the tumor suppressor gene PTEN. We observed complete loss or very faint PTEN expression in 10 of $19(53 \%)$ of tested tumor samples, highlighting the therapeutic target potential of the PI3K/AKT/mTOR pathway. Indeed activation of the mTOR signaling has been demonstrated in HCC where immunohistochemical analysis shows significantly elevated expression of p-mTOR in the sinusoidal endothelial cells of HCC tissue samples when comparison with non-cancerous tissue such as normal liver, cirrhotic nodules, or hepatic adenomas.[17, 18] Indeed, preclinical murine models of HCC have demonstrated a greater degree of carcinoma growth inhibition with the combination of bevacizumab plus rapamycin treatment rather than monotherapy with either agent.[19] Furthermore, mTOR inhibition with rapamycin has been explored in the context of decreased post-transplant tumor recurrence in patients receiving sirolimus for immunosuppression.[20] Another metaanalysis investigated 2950 patients with resectable HCC who underwent a liver transplantation; among these patients, those who were placed on a sirolimus-based regimen for immunosuppression had a decreased risk for disease recurrence and an improvement in their overall survival, highlighting the significant dual role of mTOR inhibitors in this selected population.[21]

Another emerging molecular aberration is mutations in the TP53 gene encoding the tumor suppressor protein P53. Polymorphisms particularly in codon 72 of TP53 have been associated with increased risk for multiple solid malignancies including high-grade serous ovarian and pancreatic adenocarcinoma.[22, 23]

In one series, TP53 mutations have been associated with recurrence of $\mathrm{HCC}$; indeed, 16 of 33 cases (49\%) of recurrent $\mathrm{HCC}$ were observed to harbor the P53 mutation; additionally patients whose tumor had the P53 mutation had a faster time to recurrence after surgical resection, further supporting this mutation's role in carcinogenesis. [24]

Our analysis demonstrates several limitations. First, a selection bias exists within our patient population given that the patients presented with metastatic disease and were heavily pretreated prior to the initiation of Phase I therapy. Second, a significant number of patients (39 of 100 referred patients, 39\%) were not enrolled on a phase I trial for the most part due to poor overall health and performance status. Finally, mutational analysis was performed in a small subset of patients due to the lack of 
tissue availability for testing.

Overall, the poor outcomes of advanced HCC patients emphasize the need for new approaches. Therapeutic strategies on clinical trials with inhibitor of angiogenesis and multiple kinases merit further exploration in phase II trials.

\section{PATIENTS AND METHODS}

Of 3614 patients evaluated in the Clinical Center for Targeted Therapy (Phase I Clinical Trials Program) at the M.D. Anderson Cancer Center from November 2004 onwards, we identified 100 consecutive patients with HCC. After review of baseline clinical, laboratory, radiologic and pathologic data during the initial consultation, patients were enrolled on a phase I trial based on scientific rationale and protocol availability. After initiation of an investigational therapy, patients were evaluated at 2- to 4- week intervals, based on the specific protocol, with a history, physical examination, comprehensive series of laboratory tests, and assessments of toxicity and compliance. Restaging scans were done every 6-8 weeks, depending on the protocol.

Specific eligibility criteria varied by protocol; however, common criteria for participation in most phase I clinical trials included presence of metastatic or unresectable disease, measurable disease per Response Evaluation Criteria in Solid Tumors (RECIST), and Eastern Cooperative Oncology Group (ECOG) performance status (PS) of 0-1 (although a PS of 2 is acceptable for certain investigator-initiated studies). This study and all clinical trials were approved by the Institutional Review Board.

\section{Endpoints and statistical methods}

The statistical analysis was performed by our biostatistician (K.R.H.). Descriptive statistics summarized the patients' characteristics. Cox proportional hazards regression analysis was used to examine the association between progression free survival (PFS) and overall survival (OS) since beginning phase I therapy and the following variables measured at the time of initial Phase I consultation: age at diagnosis, gender, ECOG PS, etiology of liver disease (alcohol abuse, hepatitis B and $\mathrm{C}$, fatty liver disease, etc.), extent of liver disease (cirrhosis, portal hypertension, portal vein thrombosis), number of metastatic sites, number of prior therapies, hemoglobin, lactate dehydrogenase (LDH), platelet count, history of thromboembolism, total bilirubin, aspartate aminotransferase (AST), alanine aminotransferase (ALT), alkaline phosphatase (ALK), serum sodium, serum creatinine, tumor markers (specifically alpha-fetoprotein), and serum albumin.

Best response was assessed using RECIST every
2 cycles (6-8 weeks) of therapy as per the protocol.[12] Partial response (PR) was defined as a $>30 \%$ decrease in the sum of the longest diameter of target lesions, excluding complete disappearance of disease (complete response, $\mathrm{CR}$ ) and progressive disease (PD) was a $>20 \%$ increase. Stable disease (SD) was defined as changes that did not meet the criteria for a PR or PD. Waterfall plot analysis according to RECIST is used to illustrate response.

Overall survival is defined as the date of enrollment on a Phase I trial until death from any cause or date of last follow-up. PFS is defined as the time from first day of treatment on a phase I trial to date off study of last phase I trial due to disease progression or death, not toxicity. Patients still alive at time of survival analysis and free of progression at time of PFS analysis were censored at time of last follow-up. Toxicities were assessed using the National Cancer Institute Common Terminology Criteria for Adverse Events, version per the protocol. A $P$-value $<.05$ was considered statistically significant. Statistical analysis was performed using S-PLUS ${ }^{\circledR} 8.0$ for Windows (Insightful Corp.).

\section{CONFLICTS OF INTEREST}

Razelle Kurzrock (Honoraria and research funding from Roche, Genentech, and AstraZeneca); Filip Janku (Research funding from Novartis). All other authors have no disclosures.

\section{Editorial note}

This paper has been accepted based in part on peerreview conducted by another journal and the authors' response and revisions as well as expedited peer-review in Oncotarget.

\section{FUNDING}

NIH/NCI under award number P30CA016672 Cancer Center Support Grant (CCSG).

\section{REFERENCES}

1. Lai EC, Fan ST, Lo CM, Chu KM, Liu CL and Wong J. Hepatic resection for hepatocellular carcinoma. An audit of 343 patients. Annals of surgery. 1995; 221:291-298.

2. Mazzaferro V, Regalia E, Doci R, Andreola S, Pulvirenti A, Bozzetti F, Montalto F, Ammatuna M, Morabito A and Gennari L. Liver transplantation for the treatment of small hepatocellular carcinomas in patients with cirrhosis. The New England journal of medicine. 1996; 334:693-699.

3. Gouillat C, Manganas D, Saguier G, Duque-Campos R and Berard P. Resection of hepatocellular carcinoma in cirrhotic patients: longterm results of a prospective study. Journal of the American College of Surgeons. 1999; 189:282-290. 
4. Cheng AL, Kang YK, Chen Z, Tsao CJ, Qin S, Kim JS, Luo R, Feng J, Ye S, Yang TS, Xu J, Sun Y, Liang H, Liu J, Wang J, Tak WY, et al. Efficacy and safety of sorafenib in patients in the Asia-Pacific region with advanced hepatocellular carcinoma: a phase III randomised, doubleblind, placebo-controlled trial. The lancet oncology. 2009; 10:25-34.

5. Keating GM and Santoro A. Sorafenib: a review of its use in advanced hepatocellular carcinoma. Drugs. 2009; 69:223240 .

6. Llovet JM, Ricci S, Mazzaferro V, Hilgard P, Gane E, Blanc JF, de Oliveira AC, Santoro A, Raoul JL, Forner A, Schwartz M, Porta C, Zeuzem S, Bolondi L, Greten TF, Galle PR, et al. Sorafenib in advanced hepatocellular carcinoma. The New England journal of medicine. 2008; 359:378-390.

7. Llovet JM, Real MI, Montana X, Planas R, Coll S, Aponte J, Ayuso C, Sala M, Muchart J, Sola R, Rodes J and Bruix J. Arterial embolisation or chemoembolisation versus symptomatic treatment in patients with unresectable hepatocellular carcinoma: a randomised controlled trial. Lancet. 2002; 359:1734-1739.

8. Lo CM, Ngan H, Tso WK, Liu CL, Lam CM, Poon RT, Fan ST and Wong J. Randomized controlled trial of transarterial lipiodol chemoembolization for unresectable hepatocellular carcinoma. Hepatology. 2002; 35:1164-1171.

9. Salem R, Lewandowski RJ, Mulcahy MF, Riaz A, Ryu RK, Ibrahim S, Atassi B, Baker T, Gates V, Miller FH, Sato KT, Wang E, Gupta R, Benson AB, Newman SB, Omary $\mathrm{RA}$, et al. Radioembolization for hepatocellular carcinoma using Yttrium-90 microspheres: a comprehensive report of long-term outcomes. Gastroenterology. 2010; 138:52-64.

10. Memon K, Lewandowski RJ, Riaz A and Salem R. Yttrium 90 microspheres for the treatment of hepatocellular carcinoma. Recent results in cancer research Fortschritte der Krebsforschung Progres dans les recherches sur le cancer. 2013; 190:207-224.

11. Mazzaferro V, Sposito C, Bhoori S, Romito R, Chiesa C, Morosi C, Maccauro M, Marchiano A, Bongini M, Lanocita R, Civelli E, Bombardieri E, Camerini T and Spreafico C. Yttrium(90) radioembolization for intermediate-advanced hepatocarcinoma: A phase II study. Hepatology. 2012.

12. Therasse P, Arbuck SG, Eisenhauer EA, Wanders J, Kaplan RS, Rubinstein L, Verweij J, Van Glabbeke M, van Oosterom AT, Christian MC and Gwyther SG. New guidelines to evaluate the response to treatment in solid tumors. European Organization for Research and Treatment of Cancer, National Cancer Institute of the United States, National Cancer Institute of Canada. Journal of the National Cancer Institute. 2000; 92:205-216.

13. Benson AB, 3rd, Abrams TA, Ben-Josef E, Bloomston PM, Botha JF, Clary BM, Covey A, Curley SA, D’Angelica MI, Davila R, Ensminger WD, Gibbs JF, Laheru D, Malafa MP, Marrero J, Meranze SG, et al. NCCN clinical practice guidelines in oncology: hepatobiliary cancers. J Natl Compr
Canc Netw. 2009; 7:350-391.

14. Salem R, Lewandowski RJ, Kulik L, Wang E, Riaz A, Ryu RK, Sato KT, Gupta R, Nikolaidis P, Miller FH, Yaghmai V, Ibrahim SM, Senthilnathan S, Baker T, Gates VL, Atassi B, et al. Radioembolization results in longer time-to-progression and reduced toxicity compared with chemoembolization in patients with hepatocellular carcinoma. Gastroenterology. 2011; 140:497-507 e492.

15. Naing A and Kurzrock R. Chemotherapy resistance and retreatment: a dogma revisited. Clinical colorectal cancer. 2010; 9:E1-4.

16. Cara S and Tannock IF. Retreatment of patients with the same chemotherapy: implications for clinical mechanisms of drug resistance. Annals of oncology : official journal of the European Society for Medical Oncology / ESMO. 2001; 12:23-27.

17. Subbiah IM, Brown RE and Fallon MB. Activation of the mammalian target of rapamycin signaling pathway in hepatocellular carcinoma. Hepatology. 2010; 52:1524.

18. Li W, Tan D, Zhang Z, Liang JJ and Brown RE. Activation of Akt-mTOR-p70S6K pathway in angiogenesis in hepatocellular carcinoma. Oncology reports. 2008; 20:713719.

19. Huynh H, Chow PK, Palanisamy N, Salto-Tellez M, Goh BC, Lee CK, Somani A, Lee HS, Kalpana R, Yu K, Tan PH, Wu J, Soong R, Lee MH, Hor H, Soo KC, et al. Bevacizumab and rapamycin induce growth suppression in mouse models of hepatocellular carcinoma. Journal of hepatology. 2008; 49:52-60.

20. Toso C, Merani S, Bigam DL, Shapiro AM and Kneteman NM. Sirolimus-based immunosuppression is associated with increased survival after liver transplantation for hepatocellular carcinoma. Hepatology. 2010; 51:1237-1243.

21. Liang W, Wang D, Ling X, Kao AA, Kong Y, Shang Y, Guo Z and He X. Sirolimus-based immunosuppression in liver transplantation for hepatocellular carcinoma: a metaanalysis. Liver Transpl. 2012; 18:62-69.

22. Sonoyama T, Sakai A, Mita Y, Yasuda Y, Kawamoto H, Yagi T, Yoshioka M, Mimura T, Nakachi K, Ouchida M, Yamamoto K and Shimizu K. TP53 codon 72 polymorphism is associated with pancreatic cancer risk in males, smokers and drinkers. Mol Med Report. 2011; 4:489-495.

23. Petitjean A, Mathe E, Kato S, Ishioka C, Tavtigian SV, Hainaut $\mathrm{P}$ and Olivier M. Impact of mutant p53 functional properties on TP53 mutation patterns and tumor phenotype: lessons from recent developments in the IARC TP53 database. Hum Mutat. 2007; 28:622-629.

24. Chen GG, Merchant JL, Lai PB, Ho RL, Hu X, Okada M, Huang SF, Chui AK, Law DJ, Li YG, Lau WY and Li AK. Mutation of $\mathrm{p} 53$ in recurrent hepatocellular carcinoma and its association with the expression of ZBP-89. Am J Pathol. 2003; 162:1823-1829. 